2012-4

\title{
Aircraft Route Optimization for Heterogeneous Formation Flight
}

Jia Xu

Stanford University

Andrew Ning

Brigham Young University, aning@byu.edu

Geoffrey Bower

Stanford University

Ilan Kroo

Stanford University

Follow this and additional works at: https://scholarsarchive.byu.edu/facpub

Part of the Mechanical Engineering Commons

\section{Original Publication Citation}

Xu, J., Ning, A., Bower, G., and Kroo, I., "Aircraft Route Optimization for Heterogeneous Formation Flight," 53rd AIAA Structures, Structural Dynamics and Materials Conference, Honolulu, Hawaii, Apr. 2012. doi:10.2514/6.2012-1524

\section{BYU ScholarsArchive Citation}

Xu, Jia; Ning, Andrew; Bower, Geoffrey; and Kroo, Ilan, "Aircraft Route Optimization for Heterogeneous Formation Flight" (2012). Faculty Publications. 1749.

https://scholarsarchive.byu.edu/facpub/1749

This Conference Paper is brought to you for free and open access by BYU ScholarsArchive. It has been accepted for inclusion in Faculty Publications by an authorized administrator of BYU ScholarsArchive. For more information, please contact ellen_amatangelo@byu.edu. 


\title{
Aircraft Route Optimization for Heterogeneous Formation Flight
}

\author{
Jia Xu*, S. Andrew Ning ${ }^{\dagger}$, Geoffrey Bower ${ }^{\ddagger}$ \\ and Ilan Kroo $\$$ \\ Stanford University, Stanford, CA, 94305, U.S.A
}

We examine the value of extended formation flight in the context of airline operations. The study is based on a bi-level route optimization framework. The aircraft performance model includes the effects of rolled-up wakes, formation heterogeneity, and formationinduced compressibility effects. We incorporate airline economics to understand the tradeoff between speed and drag savings in the context of formation flight. The application of formation flight to a representative South African Airlines (SAA) long-haul route network can reduce fuel burn by over $4.6 \%$ or reduce direct operating cost by $2.1 \%$. The savings increase to $6.8 \%$ in fuel or $2.4 \%$ in cost when we consider a large-scale Star Alliance transatlantic route network. Finally, the impact of compressibility on total fleet fuel and cost metrics is shown to be insignificant.

\section{Nomenclature}

$\boldsymbol{h}_{\boldsymbol{r} \boldsymbol{i}} \quad$ Reserve mission altitude states

$\boldsymbol{M}_{\boldsymbol{r} \boldsymbol{i}} \quad$ Reserve mission Mach number states

$\boldsymbol{W}_{\boldsymbol{r} \boldsymbol{i}} \quad$ Reserve mission weight states

$\Delta x \quad$ Longitudinal separation between the aircraft in the formation

$\Delta y \quad$ Lateral separation between the aircraft in the formation

$\Delta z \quad$ Vertical separation between the aircraft in the formation

$\bar{v}_{i j} \quad$ Average solo speed of aircraft $i$ and $j$

$\bar{v}_{i} \quad$ Solo speed of aircraft $i$

$\bar{v}_{j} \quad$ Solo speed of aircraft $j$

$A_{\text {three }} \quad$ Binary matrix indicating which flight are represented by each three aircraft formation

$A_{t w o} \quad$ Binary matrix indicating which flights are represented by each two aircraft formation

$A R \quad$ Aspect ratio

$b \quad$ Aircraft wing span

$b_{0} \quad$ Initial spacing between a vortex pair

$C_{i} \quad$ The $i^{\text {th }}$ network optimization constraint

$C_{L} \quad$ Aircraft lift coefficient

$D \quad$ Drag

DOC Direct operating cost

$J_{c} \quad$ Cost objective

$J_{f} \quad$ Fuel burn objective

$J_{\text {network }} \quad$ Network optimization objective function

Jsolo Optimal fuel burn or cost for each solo route from mission optimization

JthreeAC Optimal fuel burn or cost for each three aircraft formation from mission optimization JtwoAC Optimal fuel burn or cost for each two aircraft formation from mission optimization

*Ph.D. Department of Aeronautics \& Astronautics. Student Member AIAA

${ }^{\dagger}$ Ph.D. Department of Aeronautics \& Astronautics. Student Member AIAA

${ }^{\ddagger}$ Ph.D. Department of Aeronautics \& Astronautics. Student Member AIAA

$\S$ Professor, Department of Aeronautics \& Astronautics. Fellow AIAA 


\begin{tabular}{|c|c|}
\hline$N_{1}$ & Number of aircraft, also equal to the number of solo missions \\
\hline$N_{2}$ & Number of candidate two aircraft formations \\
\hline$N_{3}$ & Number of candidate three aircraft formations \\
\hline$n_{a}$ & Number of aircraft in a formation \\
\hline$q_{\infty}$ & Freestream dynamic pressure \\
\hline$r$ & Radial position \\
\hline$r_{i}$ & Distance between the arrival and departure airports of aircraft $i$ \\
\hline$r_{k}$ & Aircraft range over segment k \\
\hline$R F$ & Range factor \\
\hline$S_{\text {ref }}$ & Aircraft reference area \\
\hline solo $_{i}$ & Binary variable indicating whether or not solo mission $i$ is flown \\
\hline$T$ & Available thrust \\
\hline$t_{a}$ & Scheduled arrival time \\
\hline three $A C_{i}$ & Binary variable indicating whether or not three aircraft formation mission $i$ is flown \\
\hline$T S F C$ & Thrust specific fuel consumption \\
\hline$t w o A C_{i}$ & Binary variable indicating whether or not two aircraft formation mission $i$ is flown \\
\hline$U_{\infty}$ & Freestream velocity \\
\hline$V_{\theta}$ & Tangential velocity \\
\hline$V_{n}$ & Normalwash \\
\hline$W_{f}$ & Fuel burn \\
\hline$h_{k i}$ & Altitude for flight state $k$ for aircraft $i$ \\
\hline$l^{l a t} t_{k}$ & Latitude for flight state $k$ \\
\hline $\operatorname{lon}_{k}$ & Longitude for flight state $k$ \\
\hline$M_{k i}$ & Mach number for flight state $k$ for aircraft $i$ \\
\hline$r_{a i j}$ & Distance between the arrival airports of aircraft $i$ and $j$ in the same formation \\
\hline$r_{d i j}$ & Distance between the departure airports of aircraft $i$ and $j$ in the same formation \\
\hline$r_{k_{r}}$ & Aircraft range over reserve segment k \\
\hline$t_{a i}$ & Arrival time for aircraft $i$ \\
\hline$t_{d i}$ & Departure time for aircraft $i$ \\
\hline$t_{k i}$ & Time of flight state $k$ for aircraft $i$ \\
\hline$W_{c i}$ & Climb fuel burn for aircraft $i$ \\
\hline$W_{f_{i}}$ & Total fuel burn for aircraft $i$ \\
\hline$W_{k i}$ & Weight for flight state $k$ for aircraft $i$ \\
\hline \multicolumn{2}{|l|}{ Symbols } \\
\hline$\Gamma$ & Circulation \\
\hline$\rho_{\infty}$ & Freestream air density \\
\hline$\Delta \phi$ & Maximum allowed arrival and departure azimuth difference between aircraft in the same formation \\
\hline$\phi_{a}$ & Arrival azimuth \\
\hline$\phi_{d}$ & Departure azimuth \\
\hline
\end{tabular}

\section{Introduction}

Numerous studies have examined the aerodynamic benefits of close formation flight through both numerical and experimental means. ${ }^{1-8}$ The results agree that formation flight has the potential to significantly reduce aircraft induced drag. Our recent work examines a potentially safer approach to flying in formation, which we call extended formation flight. ${ }^{9}$ In contrast to close formation flight, extended formations separate aircraft with streamwise spacing of 5-40 wingspans. The increased longitudinal separation can render formation flight more compatible with commercial service.

Advanced vehicle-level technologies like natural laminar flow surfaces, blended wing body configurations, and open-rotor engines can only be deployed on new aircraft or on existing designs after significant retrofits. Decades may separate the initial design of a new aircraft from production and fleet-wide adoption. Formation flight on the other hand can potentially be applied to existing aircraft to realize significant fuel efficiency gains.

A key component of formation flight is the mission design problem. Bower ${ }^{10,11}$ demonstrated a method to optimize a network flight schedule using formation flight to minimize fuel consumption. The first level 
optimizes the mission for a candidate set of aircraft, while the second level optimizes the network by selecting the best schedule amongst the possible combinations of formation and solo missions. The optimization architecture is illustrated in Fig. 1. The input is the aircraft route network and flight schedules. The output is a consistent set of missions that minimize some formation-level objective function such as fuel burn or direct operating cost. Each mission optimization problem is smooth and is solved using a fast continuous domain optimization algorithm. The outer-loop network optimization is posed as an integer-programming problem that returns the best schedule given a set of optimized formation and solo missions.

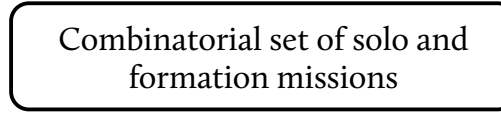

Heuristic search to eliminate

"bad" missions

n candidate missions

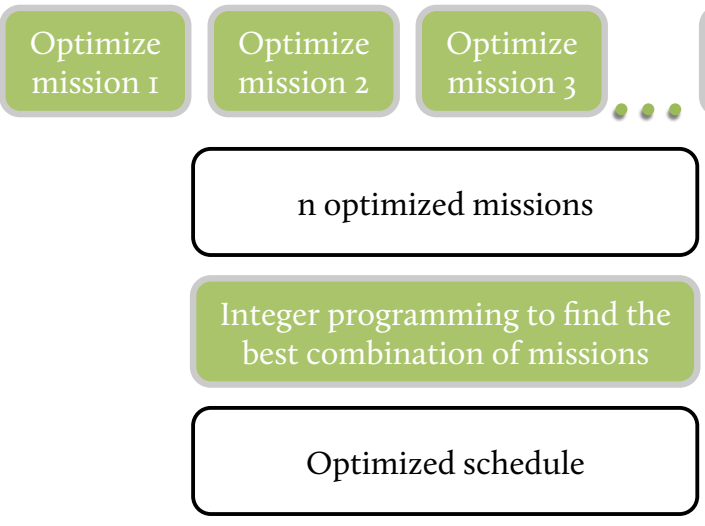

Optimize

mission $\mathrm{n}$

Figure 1. A flowchart illustrating the architecture of the bi-level optimization.

We extend the bi-level network optimization framework to addresses several yet unresolved questions related to the economic and operational viability of formation flight. We optimize the 4-D trajectory of aircraft in formations - including the previous omitted problems of altitude scheduling. The analysis also incorporates a heterogeneous aircraft formation drag model based on a rolled-up wake appropriate for extended formations. ${ }^{9}$ We include a cost-sensitive objective function to study the economic impact of formation flight. Finally, we examine the impact of compressibility constraints on formation flight performance.

\section{Heuristic Formation Search}

For a schedule with $n$ direct flights there are $n(n-1)$ possible 2-aircraft and $n(n-1)(n-2)$ possible 3-aircraft formations. The combinatorial growth of the set of all possible formations renders the scheduling problem NP-hard. We recognize however, that only a small subset of the set of all possible formations is viable. For example, aircraft that have to fly to destinations in the exact opposite directions are not good candidates for formation flight. To render the formation optimization problem tractable we develop a series of pragmatic design heuristics to find good "candidate" formations for mission optimization.

The heuristics are based on the spatial and temporal separations between aircraft in formation and the geometry of the formation routes. First, the differences in the departure and arrival azimuth between aircraft $i$ and $j$ in the same formation must be less than $\Delta \phi_{i j}$. The azimuth rule in Eq. (1) eliminates formations that contain aircraft traveling in significantly different directions.

$$
\begin{array}{r}
\left|\phi_{a_{i}}-\phi_{a_{j}}\right|<\Delta \phi_{i j} \\
\left|\phi_{d i}-\phi_{d_{j}}\right|<\Delta \phi_{i j}
\end{array}
$$

We also limit the separation between the departure and arrival airports of the candidate formation. It is 
useful to introduce the concept of a modified distance, $r_{m}$ in Eq. (2), which combines the distance between airports with schedule differences. The modified departure distance between aircraft in a candidate formation are required to fall below some threshold distance $r_{\max }$ (similarly for the modified arrival distance).

$$
\begin{aligned}
& r_{d i j}+\bar{v}_{i j}\left(\left|t_{d i}-t_{d j}\right|-2 \Delta t\right)<r_{\text {max }} \\
& r_{a i j}+\bar{v}_{i j}\left(\left|t_{a i}-t_{a j}\right|-2 \Delta t\right)<r_{\max }
\end{aligned}
$$

Where the average speed $\bar{v}_{i j}$ is defined as:

$$
\bar{v}_{i j}=\frac{\bar{v}_{i}+\bar{v}_{j}}{2}
$$

and the $\bar{v}_{i}$ is defined by the scheduled solo route distance and flight time. A similar heuristic requires that the sum of departure and arrival distances $r_{d i j}$ and $r_{a i j}$ should be small relative to the sum of the route distances $r_{i}$ and $r_{j}$. The geometric constraint on the "aspect ratio" of the formation defined in Eq. (3) favors aircraft formations that have clustered origins and destinations with long distances between the origin-destination pairs. Experimentation shows that a $\kappa_{r}$ of 0.3 is appropriate for the route networks in this study.

$$
\frac{r_{d i j}+r_{a i j}}{r_{i}+r_{j}}<\kappa_{r}
$$

We require the flight times of aircraft in formations to overlap. The degree of overlap determines the potential for fuel savings and the relative cost of getting in and out of formation. It would be impossible, for example, for two aircraft to fly in the same formation if one lands before the other can take off. The factor $\kappa_{t}$ is defined as the ratio between the overlap time and the total scheduled flight time for aircraft $i$ and $j$ :

$$
\frac{\min \left(t_{a i}, t_{a_{j}}\right)-\max \left(t_{d i}, t_{d j}\right)+2 \Delta t}{\left(t_{a i}-t_{d i}\right)+\left(t_{a j}-t_{d j}\right)}>\kappa_{t}
$$

The overlap time ratio includes the effect of the schedule flexibility $\Delta t$. We use a $\kappa_{t}$ between $0.4-0.5$ in subsequent design studies depending on the scale of the problem. In formations where the aircraft flight times are very different, the longer flight tends to dominate. This could eliminate viable short-haul routes. However, the savings from these short-range missions are modest and should not significantly alter the system-level result.

Finally, we conduct sensitivity studies on the individual heuristics using reference schedules to ensure that they do not remove promising formations for optimization. In each case the heuristics set greatly reduced the number of candidate formations to be optimized without changing the final optimized schedules.

\section{Mission Optimization}

The next step is to optimize each candidate formation identified by the heuristic search. The potentially large numbers of candidate formations, combined with the large number of design variables makes the mission optimizations the most expensive part of the design problem. We choose a gradient-based optimizer (MATLAB's fmincon) to efficiently solve the large-scale problem. The basic structure of a formation mission is illustrated by Fig. 3 .

We optimize the 4-D trajectory of each aircraft in the formation. We divide the aircraft mission into three cruise segments shown in Fig. 2. In a solo mission, the division of the mission into segments approximates step climbs. In a formation mission the middle segment is flown in formation. A representative 3-aircraft formation mission is shown schematically in Fig. 3. The departure and destination pairs in green, red, and blue define each mission. Each node represents a flight state and is defined by longitude, latitude, and altitude. Each segment represents a great circle track. The departure and arrival coordinates are defined by schedule; the rendezvous and separation points at nodes 2 and 3 are subject to optimization.

The aircraft cruise altitude, weight, and Mach number are defined at each flight state:

$$
\begin{aligned}
\boldsymbol{W}_{\boldsymbol{i}} & =\left[W_{1 i}, W_{2 i}, W_{3 i}, W_{4 i}\right] \\
\boldsymbol{h}_{\boldsymbol{i}} & =\left[h_{1 i}, h_{2 i}, h_{3 i}, h_{4 i}\right] \\
\boldsymbol{M}_{\boldsymbol{i}} & =\left[M_{1 i}, M_{2 i}, M_{3 i}, M_{4 i}\right]
\end{aligned}
$$




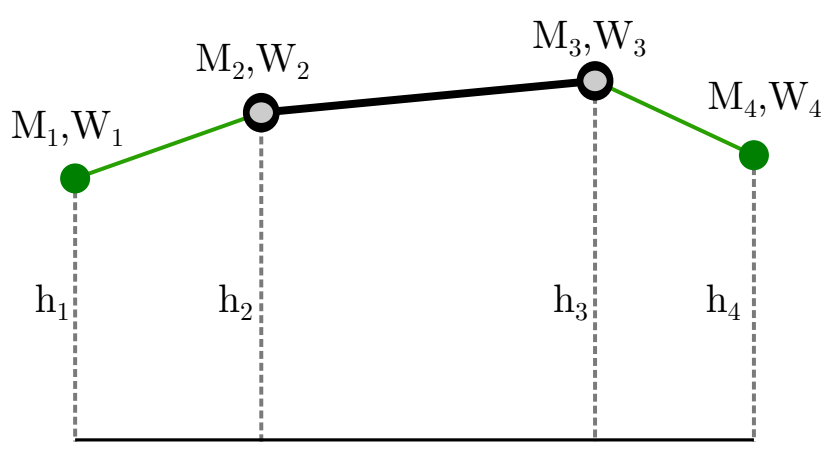

Figure 2. The solo mission parameterization.

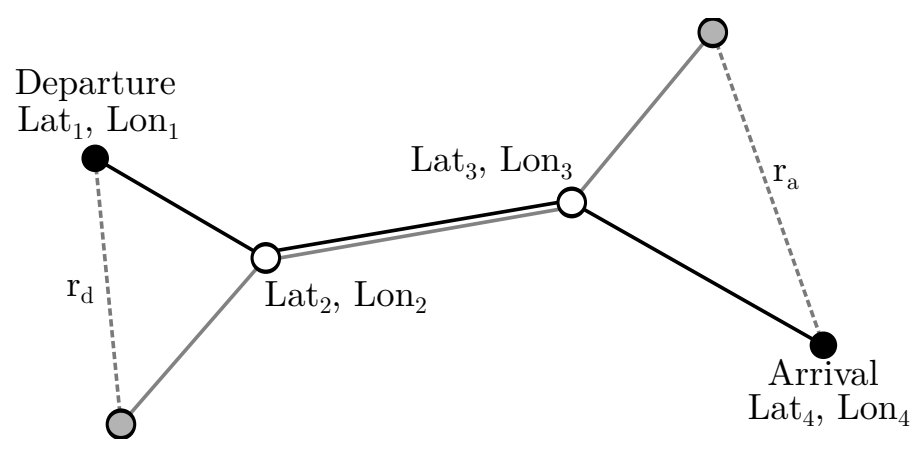

Figure 3. The structure of a three-aircraft formation mission. The black segment is flown in formation

Aircraft in the same formation share the same Mach numbers and altitudes at the beginning to the end of the formation segment:

$$
\begin{aligned}
h_{2 i} & =h_{2} \\
h_{3 i} & =h_{3} \\
M_{2 i} & =M_{2} \\
M_{3 i} & =M_{3}
\end{aligned}
$$

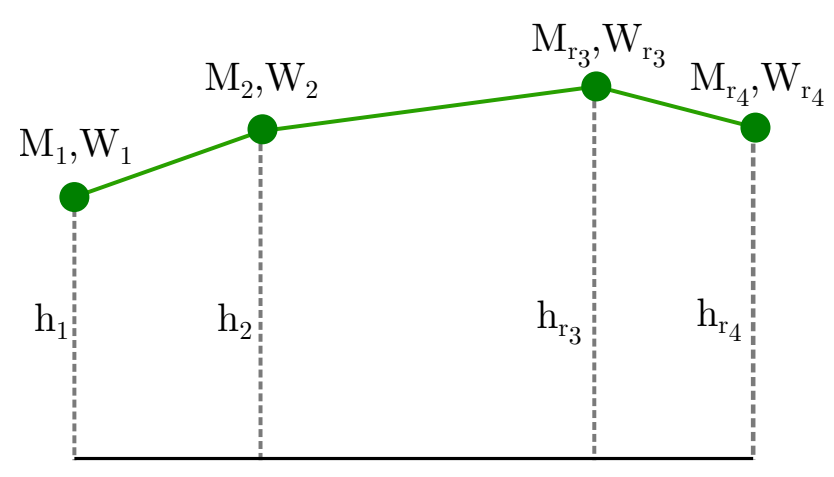

Figure 4. The reserve mission parameterization.

Aircraft in formation must also carry sufficient fuel to complete the reserve mission illustrated in Fig. 4. The mission represents the worst-case scenario where an aircraft is committed to the formation route but fails to achieve any drag savings. The reserve missions are defined with the additional parameters in Section III. Note that aircraft can alter their altitude and speed to more efficiently fly the reserve missions. The reserve 
mission is posed as a constraint: the fuel burn objective still comes from the formation cruise performance.

$$
\begin{aligned}
\boldsymbol{W}_{\boldsymbol{r i}} & =\left[W_{3 r i}, W_{4 r i}\right] \\
\boldsymbol{h}_{\boldsymbol{r i}} & =\left[h_{3 r i}, h_{4 r i}\right] \\
\boldsymbol{M}_{\boldsymbol{r} \boldsymbol{i}} & =\left[M_{3 r i}, M_{4 r i}\right]
\end{aligned}
$$

\section{A. Objective}

The objective of the mission optimization is to minimize either the fuel burn $\left(J_{f_{i}}\right)$ or the direct operating cost (DOC) $\left(J_{c i}\right)$ of a solo or formation mission:

$$
\begin{aligned}
J_{f_{i}} & =\min \sum_{i=1}^{n_{a}} W_{f_{i}}=\min \sum_{i=1}^{n_{a}}\left(W_{1 i}-W_{4 i}+W_{c i}\right) \\
J_{c i} & =\min \sum_{i=1}^{n_{a}} D O C_{i}
\end{aligned}
$$

The fleet fuel burn can be written as sum of the change in weight from the beginning to the end of each mission. The landing stage is assumed to consume no additional fuel over the equivalent cruise distance. The takeoff and climb fuel burn $W_{c}$ is estimated as a function of the weight, altitude, and Mach number at the start of cruise:

$$
W_{c i}=f\left(W_{1 i}, h_{1 i}, M_{1 i}\right)
$$

The inclusion of a cost metric captures the impact of block and flight speed on airline economics. Formation flight can reduce fuel burn but can also increase block time. To get into formation aircraft have to divert from their shortest route. Moreover, a formation can only fly as fast as its slowest member. At low fuel prices the cost penalties associated with longer flights may well outweigh the savings in fuel.

We estimate the direct operating cost as the sum of costs that scale with the flight time $t_{\text {cruise }}$, the block time $t_{\text {block}}$, and the fuel burn. ${ }^{12}$ The key cost assumptions are a fuel cost of $\$ 3.3 /$ gallon, and a maintenance labor rate of $\$ 40 /$ hour. Costs that scale with the years in operation, such as aircraft depreciation and insurance, are identical for aircraft flying in and out of formations and are therefore not considered. The same is true of costs that scale with the number of flight cycles such as landing fees.

\section{B. Variables}

The design variables can be divided into the solo flight segment variables $\boldsymbol{x}_{\boldsymbol{s}}$, the formation flight segment variables $\boldsymbol{x}_{\boldsymbol{f}}$, and the reserve segment variables $\boldsymbol{x}_{\boldsymbol{r}}$. The solo mission variables are defined for each aircraft:

$$
\boldsymbol{x}_{\boldsymbol{s}}=\left[h_{1 i}, h_{4 i}, M_{1 i}, M_{4 i}, W_{1 i}, W_{2 i}, W_{3 i}, W_{4 i}, \Delta t_{d i}, \Delta t_{a i}\right]
$$

$\Delta t_{d i}$ and $\Delta t_{a i}$ are the change in departure and arrival time for aircraft $i$. Departure and arrival flexibility have a significant impact on cost since they help determine how much aircraft have to slow down to rendezvous.

The formation segment variables are defined for each formation:

$$
\boldsymbol{x}_{\boldsymbol{f}}=\left[h_{2}, h_{3}, M_{2}, M_{3}, \text { lat }_{2}, \text { lat }_{3}, \text { lon }_{2}, \text { lon }_{3}\right]
$$

The coordinate pairs $\left[l a t_{2}, l_{o n}, h_{2}\right]$ and $\left[l^{2} t_{3}, l n_{3}, h_{3}\right]$ define the shared rendezvous and separation points. Finally, the reserve segment variables are defined for each aircraft flown in formation:

$$
\boldsymbol{x}_{\boldsymbol{r}}=\left[h_{r 3 i}, h_{r 4 i}, M_{r 3 i}, M_{r 4 i}, W_{r 3 i}, W_{r 4 i}\right]
$$

\section{Constraints}

The mission optimization is subject to range, drag, time, and weight constraints defined at each flight state. A simplified performance analysis module, based on the methods contained in the Program for Aircraft Synthesis Studies (PASS) is used to obtain the constraint violations. ${ }^{13}$ The single aircraft methods in PASS 
are supplemented by the physics-based induced drag factors discussed in Section V to account for formation flight. First, each aircraft must meet range constraints over each of its flight segments $k$.

$$
d_{k}<r_{k}
$$

The aircraft must also meet a thrust margin constraint at each flight state.

$$
\frac{D}{T}<0.88
$$

We pose the analogous thrust margin and range constraints on the reserve mission in the case of formation missions. The segment distance $d_{k}$ are computed on great circle routes. We integrate for the segment range $r_{k}$ by assuming a linear variation of range factor from the start to the end of each cruise segment:

$$
r_{k}=R F_{k}-R F_{k+1}+\left(R F_{k+1}-W_{k+1} \frac{R F_{k+1}-R F_{k}}{W_{k+1}-W_{k}}\right) \ln \left(\frac{W_{k}}{W_{k+1}}\right)
$$

Where the range factor $R F$ of a flight segment is defined as:

$$
R F_{k}=\frac{v_{k}}{T S F C_{k}}\left(\frac{L}{D}\right)_{k}
$$

At flight state $k$ we trim the aircraft and obtain the wing and horizontal tail $C_{L}$. Next the inviscid component of the induced drag is computed based on assumptions of elliptical lift and a semi-empirical accounting of fuselage and horizontal tail effects. If the segment is flown in formation then the inviscid induced drag is multiplied by the formation drag reduction factor discussed in Section V. The parasite drag is computed using equivalent plate area methods with compressibility-corrected surface friction coefficient $C_{f}$ and shape factors for the fuselage, wing, and tails. ${ }^{13}$ The aircraft compressibility drag is estimated using the method of McGeer and Shevell. ${ }^{14}$ The available engine thrust and TSFC are computed using a rubberized PW2037 turbofan deck. TSFC and thrust-to-weight correction factors are used to adjust the deck to emulate the performance of more modern engines.

The aircraft departure and arrival times are constrained to lie within some $\Delta t_{d}$ and $\Delta t_{a}$ of the scheduled departure and arrival times respectively:

$$
\begin{aligned}
& t_{a i}-\Delta t_{a i}<t_{4 i}<t_{a i}+\Delta t_{a i} \\
& t_{d i}-\Delta t_{d i}<t_{1 i}-t_{c i}<t_{d i}+\Delta t_{d i}
\end{aligned}
$$

Where $t_{c}$ is an estimated climb time. The total flight time is further constrained to lie within some $\Delta t_{f}$ of the scheduled flight time:

$$
t_{a i}-t_{d i}-\Delta t_{f}<t_{4 i}-\left(t_{1 i}-t_{c}\right)<t_{a i}-t_{d i}+\Delta t_{f}
$$

Here $\Delta t_{a}$ captures the effect of schedule flexibility and flight time allowances to accommodate formation flight. The formation missions with more than one aircraft are further subject to departure, rendezvous and arrival time constraints. The rendezvous and separation time for aircraft in formation are enforced through equality constraints:

$$
\begin{aligned}
& t_{2 i}=t_{2} \\
& t_{3 i}=t_{3}
\end{aligned}
$$

Linear constraints ensure that the weight of the aircraft decreases in the course of the mission:

$$
k_{m} W_{k i}>W_{k+1_{i}}
$$

The linear weight constraints may be redundant but serve two important functions: 1) they increase the rate of convergence and 2) they remove singularities from the range calculations. We set $k_{m}$ to 0.98 to ensure that flight segments have finite length and the mission control points are distinct.

Finally, the outcome of the mission optimization is a set of individually optimal formation and solo missions. The set does not form a consistent schedule: one aircraft can appear in multiple missions. 


\section{Network Optimization}

The network optimization problem selects the optimum network schedule among combinations of the optimal solo and formation missions. The CPLEX integer-programming software is used to solve the network optimization problem. The problem is setup using the AMPL environment so that the same problem definition can be used for networks of arbitrary size. For a given problem the following parameters are defined:

1. The fuel burn or cost for each optimized solo mission (Jsolo, size $N_{1} \times 1$ )

2. The fuel burn or cost for each optimized two aircraft formation mission (JtwoAC, size $N_{2} \times 1$ )

3. The fuel burn or cost for each optimized three aircraft formation mission (Jthree $A C$, size $N_{3} \times 1$ )

4. A binary matrix indicating which flights are represented by each two aircraft formation mission $\left(A_{t w o}\right.$, size $N_{2} \times N_{1} . A_{t w o_{i, j}}=1$ if aircraft $j$ is part of formation $i$.)

5. A binary matrix indicating which flights are represented by each three aircraft formation mission $\left(A_{\text {three }}\right.$ size $N_{3} \times N_{1} . A_{\text {three }_{i, j}}=1$ if aircraft $j$ is part of formation $i$.)

The design variables are binary variables indicating which solo missions $\left(\right.$ solo $\left._{i}\right)$, two aircraft formation missions $\left(t w o A C_{i}\right)$, and three aircraft formation missions $\left(\right.$ three $\left.A C_{i}\right)$ are to be flown.

The objective function is to minimize either fuel burn or direct operating cost:

$$
J_{\text {network }}=\sum_{i=1}^{N_{1}} J_{\text {solo }_{i}} * \text { solo }_{i}+\sum_{i=1}^{N_{2}} J \text { two } A C_{i} * \text { two } A C_{i}+\sum_{i=1}^{N_{3}} J \text { three } A C_{i} *{\text { three } A C_{i}}
$$

The following constraint is applied to each of the $N_{1}$ aircraft to ensure it is either flying a solo mission, a two aircraft formation, or a three aircraft formation:

$$
C_{i}=1=\text { solo }_{i}+\sum_{j=1}^{N_{2}} A_{\text {two }_{i, j}} *{\text { two } A C_{j}}+\sum_{i=1}^{N_{3}} A_{\text {three }_{i, j}} *{\text { three } A C_{j}}
$$

The CPLEX solver uses a branch and bound method with additional heuristics to efficiently solve integerprogramming problems. The network optimization takes only about 0.3 seconds on an AMD Opteron 6172 core.

\section{Formation Aerodynamics}

Ning ${ }^{9}$ describes the aircraft wake development model used in this analysis in the previous work. This methodology uses a far-field conservation method ${ }^{15}$ to compute the rolled-up vorticity distribution of an aircraft's wake. The model is augmented with experimental data on core size,${ }^{16}$ and a viscous decay model based on LES and experimental data. ${ }^{17}$ The streamwise separation distances of the aircraft in the formation are limited to less than 40 wingspans, so only a simplified version of that wake decay model is needed. This augmented Betz approach has been shown to agree well with Navier-Stokes solutions for a variety of configurations. ${ }^{18}$

While this method is already relatively fast to evaluate, because the optimization involves evaluating the drag of thousands of formation combinations, it is desirable to further increase the speed of computation. King and Gopalarathnam ${ }^{19}$ have shown that a formation with elliptically loaded aircraft has very nearly the same induced drag as an optimally loaded formation subject to constraints on trim (for planar wings with no overlap in the wing traces). Thus, to a good approximation, each aircraft in the formation is assumed to be elliptically loaded. This significantly speeds up the computation because the tangential velocity profile induced by the wake vortices can be pre-computed (properly normalized). Fig. 5 shows an example of this self-similar velocity profile. This particular example is computed using the Augmented Betz method, however any reasonable method could be used such as a Navier-Stokes calculation or experimental data.

For an elliptically loaded wing, the spacing between the rolled-up vortices is given by

$$
b_{0}=\frac{\pi}{4} b
$$

and the total vortex circulation by

$$
\Gamma_{0}=\frac{U_{\infty} C_{L} S_{r e f}}{2 b_{0}}
$$




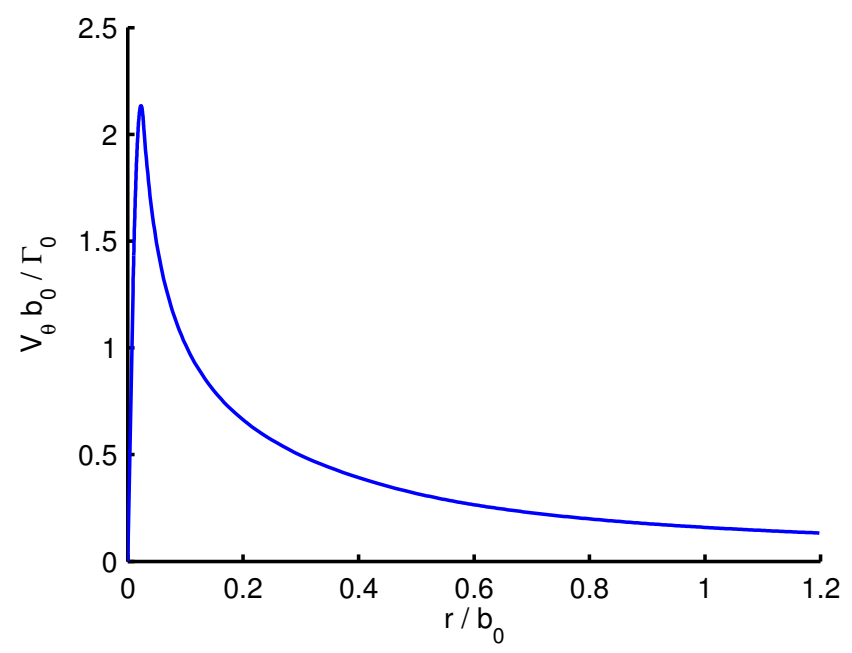

Figure 5. The tangential velocity distribution of a wake vortex as a function of distance from the vortex center (elliptically loaded wing).

With those parameters, and the non-dimensional tangential velocity profile of the rolled-up vortex, the induced velocity on a trailing aircraft from all upstream vortices can be computed. In addition, the wake is allowed to decay to account for differences in longitudinal separation. Because we restrict ourselves to only moderate separation distances (less than 40 spans), a relatively simple decay model can be used. We use the diffusion phase of a model developed by Holzäpfel ${ }^{17}$

$$
\Gamma\left(t^{*}\right)=\Gamma_{0}\left[A-\exp \left(\frac{-R^{* 2}}{\nu_{1}^{*}\left(t^{*}-T_{1}^{*}\right)}\right)\right]
$$

where $A, R^{*}, T_{1}^{*}, \nu_{1}^{*}$ are coefficients tuned from LES simulations and, $t^{*}=\Gamma_{0} /\left(2 \pi b_{0}^{2}\right) t$.

The induced drag of the trailing aircraft is then given by

$$
D_{i}=\frac{C_{L}^{2}}{\pi A R} q_{\infty} S_{r e f}+\int \rho_{\infty} V_{n} \Gamma d s
$$

where the integral is along the wing trace, and the normalwash is due only to the wake influence from upstream aircraft.

This approach still allows all the important parameters of interest to be included in the model. These parameters include the lift coefficient, thrust specific fuel consumption, wing area, span, and aircraft relative spacing (in all three directions). The induced drag can be written as:

$$
C_{d i}=f\left(\boldsymbol{C}_{\boldsymbol{L}}, \boldsymbol{T} \boldsymbol{S F C}, \boldsymbol{S}_{\boldsymbol{r e f}}, \boldsymbol{b}, \Delta x, \Delta y, \Delta z\right)
$$

Of course, specific fuel consumption of the engines does not directly affect the aircraft's induced drag. However, for formations in which the aircraft differ in size and/or engine efficiency, the order in which the aircraft fly and the type of formation ( $\mathrm{V}$, inverted- $\mathrm{V}$, echelon) affects the total fuel burn. For each set of aircraft, the analysis evaluates all potential formation combinations (2 combinations for 2-aircraft formations, and 9 for 3-aircraft formations) and chooses the arrangement with minimum fuel burn. This optimal configuration is computed at the start of the formation cruise. The configuration is not changed over the cruise segment, as benefits from rearrangement are typically minimal. ${ }^{20}$ For the subsequent designs studies we assume that the streamwise separation is 20 spans. The y and $\mathrm{z}$ offset between the wing tip and the wake are initially assumed to be 0 . Implicit is the assumption that we can accurately track the wake development in flight. Finally, we estimate the profile, compressibility, and viscous component of lift-induced drag using semi-empirical methods. ${ }^{13,14}$ 


\section{South African Airlines Study}

We optimize an airline route schedule for formation flight to quantify the system-level savings. The 31-flight South African Airway (SAA) long-haul schedule used in the study is shown in Fig. 6. The schedule includes Airbus A330-200, A340-200/300/600 and Boeing 747-400 aircraft.

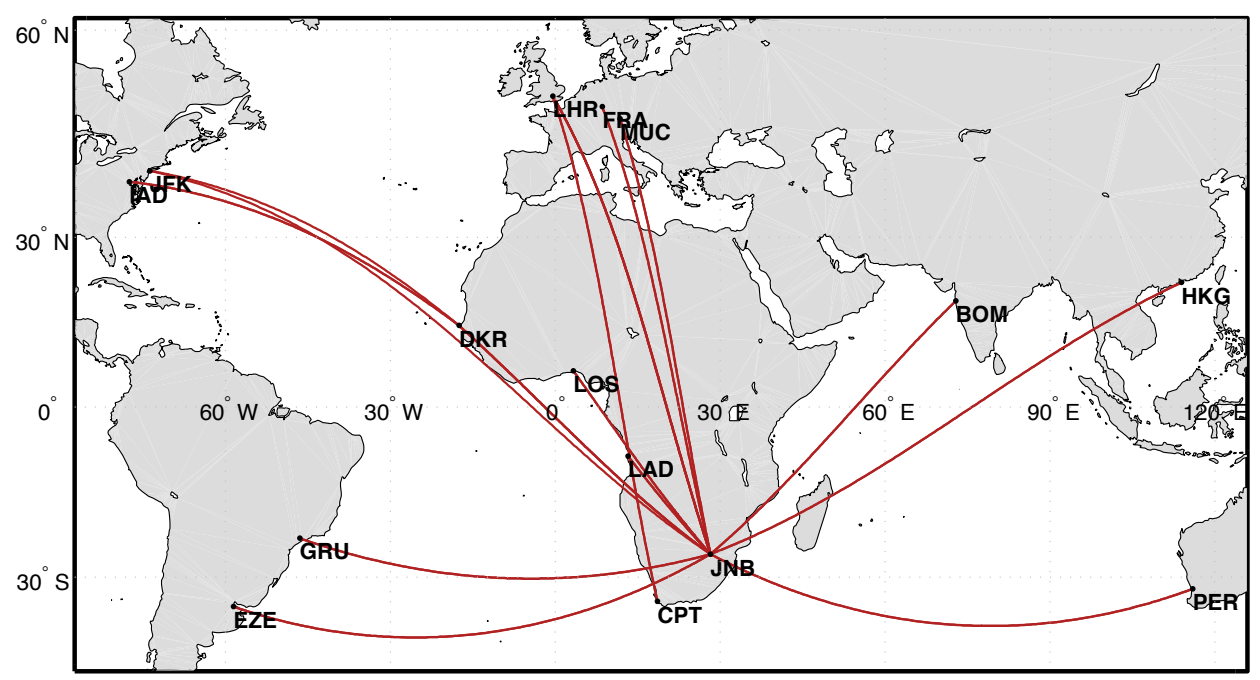

Figure 6. The SAA route network.

\begin{tabular}{ll}
\hline$\kappa_{r}$ & 0.4 \\
$\kappa_{t}$ & 0.4 \\
$r_{\text {mmax }}$ & $500 \mathrm{~nm}$ \\
$\Delta x$ & 20 spans \\
$\Delta y$ & 0 spans \\
$\Delta z$ & 0 spans \\
$\Delta \phi_{i j}$ & $120^{\circ}$ \\
$\Delta t_{a i}$ & 1 hour \\
$\Delta t_{d i}$ & 1 hour \\
\hline
\end{tabular}

Table 1. The SAA heuristic filter and formation design parameters.

The formation heuristic filter and scheduling flexibility settings are summarized in Tab. 1. Since the network is small we use relatively unrestrictive search heuristics to select the candidate formations for optimization. The optimizations are performed with a generous one-hour schedule flexibility in both the departure and arrival time.

Fig. 7 and 8 show the SAA schedule optimized for minimum fuel burn and cost respectively. The results show that the route structures of the minimum cost and minimum fuel formations are quite similar.

The results in Tab. 2 show that a formation flight schedule designed to minimize fuel burn can net a $4.6 \%$ reduction in fuel and a $1.9 \%$ reduction in cost relative to the minimum fuel solo network. Alternatively, a minimum DOC route network can expect to save $4.24 \%$ in fuel burn and $2.11 \%$ in DOC relative to the minimum DOC solo network.

Tab. 3 shows more details of the two optimized formation flight schedules. The results show that one more 2-aircraft formation is flown for the minimum fuel formation. In the minimum DOC case some $50 \%$ of the flight distance and time are flown in formation. The time and distance in formation increases to over $56 \%$ for the minimum fuel case. The results are intuitive. If the objective is to minimize fuel burn then aircraft can divert substantially from their direct great circle route to meet up in formations. However, if 


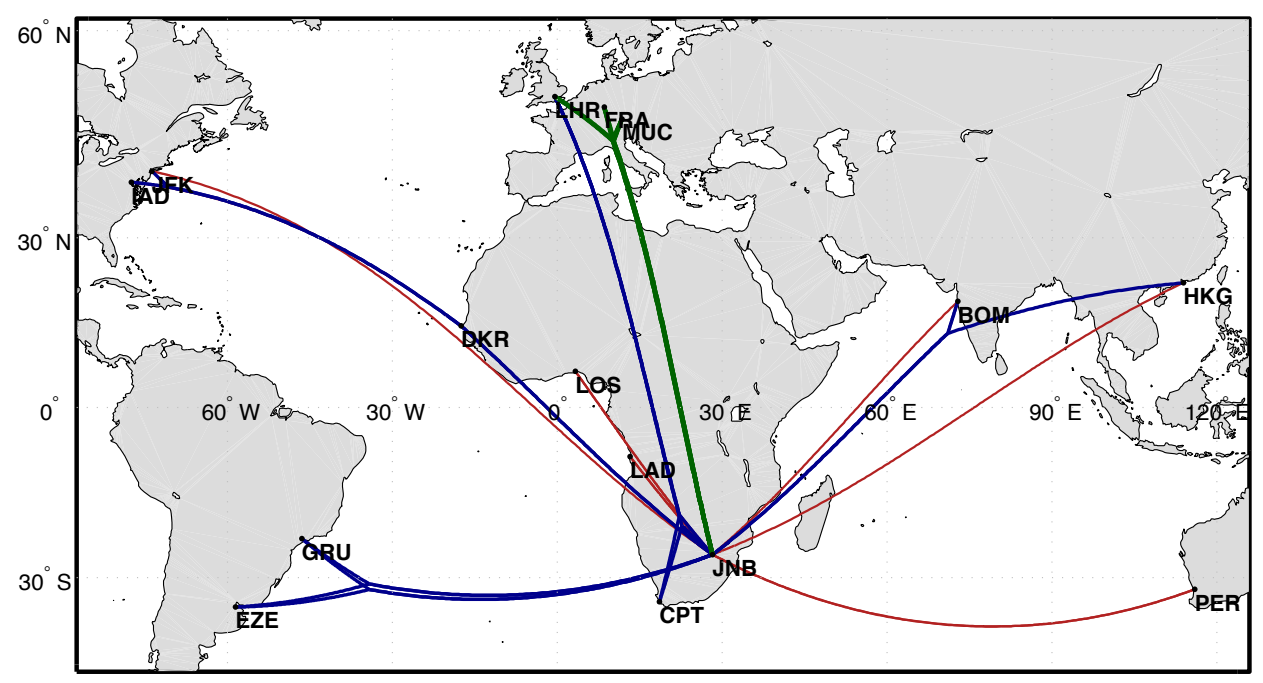

Figure 7. The SAA network optimized for minimum fuel formation flight. Two-aircraft formation segments are shown in blue. Three aircraft formations are in green.

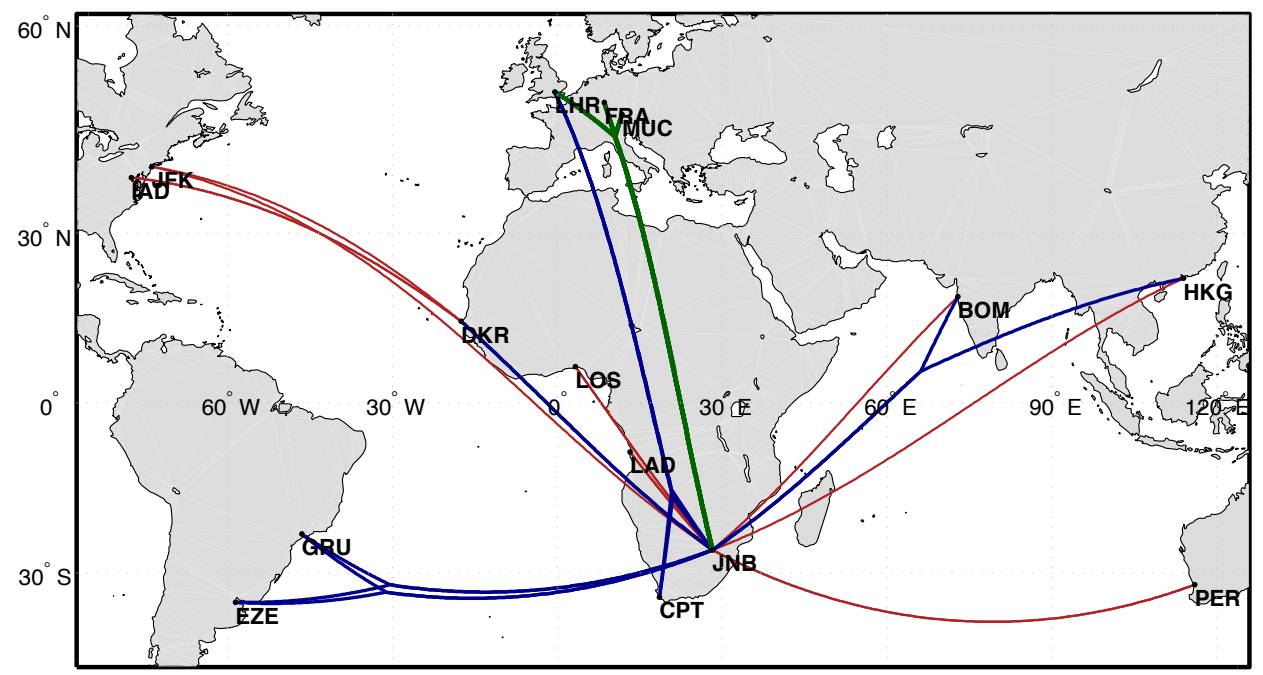

Figure 8. The SAA network optimized for minimum DOC formation flight.

\begin{tabular}{lll}
\hline & Min. DOC & Min. Fuel \\
\hline$\Delta$ Fuel & $-4.24 \%$ & $-4.64 \%$ \\
$\Delta$ Cost & $-2.11 \%$ & $-1.89 \%$ \\
$\Delta$ Time & $2.18 \%$ & $2.55 \%$ \\
$\Delta$ Distance & $0.63 \%$ & $0.84 \%$ \\
\hline
\end{tabular}

Table 2. The SAA formation flight savings. The data in the columns are referenced against the respective minimum DOC and Fuel solo networks. 


\begin{tabular}{lll}
\hline & Min. DOC & Min. Fuel \\
\hline Solo Flights & 13 & 11 \\
2-Aircraft Formations & 6 & 7 \\
3-Aircraft Formations & 2 & 2 \\
Distance in Formation & $49.7 \%$ & $56.3 \%$ \\
Time in Formation & $50.0 \%$ & $56.5 \%$ \\
Average $\Delta$ Departure Time & $32 \mathrm{~min}$ & $37 \mathrm{~min}$ \\
\hline
\end{tabular}

Table 3. SAA formation network analytics.

the routing decision is made on the basis of operating costs then the increased block and flight time to get in and out of formation can take its toll. The number of formations decreases when we switch from fuel to cost objectives. The formation rendezvous points have also shifted further toward the destination to reduce the diversion time at the cost of formation induced drag savings. The two effects decrease the total time and distance flown in formations.

The balance between cost and fuel benefits is very much defined by the cost of fuel. The optimum minimum-cost formation is a strong function of the fuel cost. Tab. 4 and 5 showcase the variations in the SAA formation savings and structures as we increase the fuel price from $\$ 2 /$ gal to $\$ 5 /$ gal. The results confirm that higher fuel prices increase the numbers of formations and the distance flown in formation. The cost savings become more significant as the fuel cost accounts for an ever-larger portion of the DOC. The impact on the formation structure is telling: at a fuel cost of $\$ 2$ per gallon only $35 \%$ of the route distance are flown in formation. This ratio increases to $55 \%$ as the cost of fuel increases to $\$ 5$ per gallon.

\begin{tabular}{lllll}
\hline & $\mathbf{\$ 2} \mathbf{\text { gal }}$ & $\mathbf{\$ 3} \mathbf{\text { gal }}$ & $\mathbf{\$ 4} /$ gal & $\mathbf{\$ 5} \mathbf{\text { gal }}$ \\
\hline$\Delta$ Fuel & $-3.13 \%$ & $-4.23 \%$ & $-4.24 \%$ & $-4.62 \%$ \\
$\Delta$ Cost & $-1.13 \%$ & $-1.71 \%$ & $-2.11 \%$ & $-2.52 \%$ \\
$\Delta$ Time & $0.96 \%$ & $2.11 \%$ & $2.18 \%$ & $2.76 \%$ \\
$\Delta$ Distance & $0.35 \%$ & $0.59 \%$ & $0.63 \%$ & $0.77 \%$ \\
\hline
\end{tabular}

Table 4. A comparison of minimum-cost SAA formation flight results at different fuel costs. The change in network-wide performance are normalized by the corresponding minimum cost solo network.

\begin{tabular}{lllll}
\hline & $\mathbf{\$ 2 / \text { gal }}$ & $\mathbf{\$ 3 / \text { gal }}$ & $\mathbf{\$ 4 / \text { gal }}$ & $\mathbf{\$ 5 / \text { gal }}$ \\
\hline Solo Flights & 19 & 13 & 13 & 11 \\
2-Aircraft Formations & 3 & 6 & 6 & 7 \\
3-Aircraft Formations & 2 & 2 & 2 & 2 \\
Distance in Formation & $34.7 \%$ & $49.1 \%$ & $49.7 \%$ & $55.6 \%$ \\
Time in Formation & $35.0 \%$ & $49.4 \%$ & $50.0 \%$ & $55.8 \%$ \\
Average $\Delta$ Departure Time (minutes) & 28 & 33 & 32 & 38 \\
\hline
\end{tabular}

Table 5. A comparison of the SAA formation flight analytics at different fuel costs.

\section{A. SAA Compressibility Effect Mitigation Study}

The drag reductions from formation flight are realized by flying trailing aircraft in the upwash of leading aircraft wake(s). However, at transonic speed the increased local angles of attack from the upwash can lead to stronger shocks and even flow separation. To cope with the compressibility effects the trailing aircraft can either slow down or fly further away from the wake to moderate. Slowing down can increase the cost. Flying further away from the wake can reduce the drag savings. We examine three compressibility mitigation strategies to the SAA route network and examine their impact on the network-wide fuel and cost 
performances. The three different combinations of aircraft-wake spacing and formation speed reductions are summarized in Tab. 6. Here $\Delta M$ is defined in terms of the allowable formation cruise Mach number, which is in turn dictated by the slowest aircraft in the formation. The $\Delta y$ separation is defined in terms of the span of the leading aircraft.

\begin{tabular}{ccc}
\hline & $\Delta M$ & $\Delta y$ \\
\hline Case 1 & $-2.5 \%$ & 0 \\
Case 2 & $-1 \%$ & $0.05 b$ \\
Case 3 & 0 & $0.10 b$ \\
\hline
\end{tabular}

Table 6. Three combinations of tip separations and speed reductions to cope with compressibility effects.

The speed reductions and lateral separations are selected based on Euler analyses of wake propagation and formation flight conducted by Ning. ${ }^{18}$ The three cases are deemed to have roughly the same compressible drag penalty as the same aircraft flying solo near their drag-divergence Mach number.

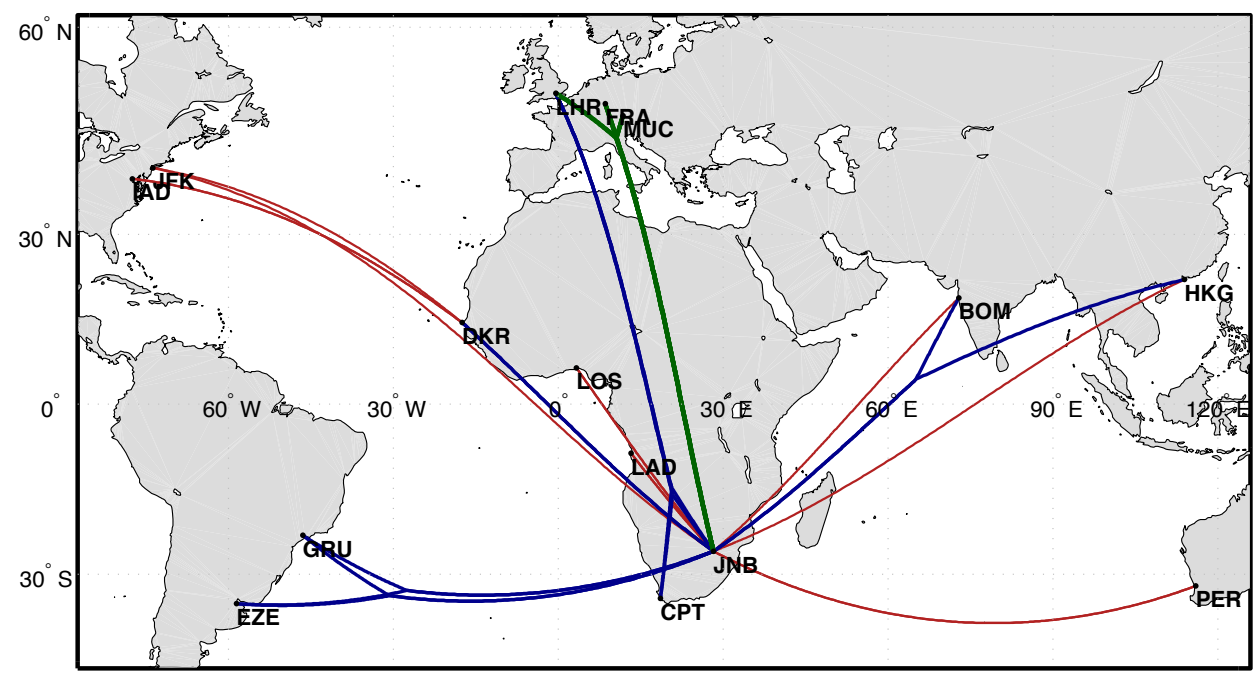

Figure 9. The minimum DOC SAA formation network with a $2.5 \%$ reduction in the maximum allowable formation cruise Mach number. Two-aircraft formation segments are shown in blue. Three aircraft formations are in green.

The compressibility design study results in Tab. 7 show that the network-wide cost and fuel burn penalties of the compressibility mitigation strategies are all small. Compressibility effects on the trailing aircraft may not therefore constitute a significant constraint on formation flight. Of the three strategies, slowing down by $2.5 \%$ has the least negative impact on cost and fuel consumption. In fact the fuel consumption is unchanged from the minimum DOC network. This is expected as slowing down can have a positive effect on fuel burn while increasing separation distance always reduces the induced drag savings.

\begin{tabular}{lllll}
\hline & Min. DOC & Case 1 & Case 2 & Case 3 \\
\hline$\Delta$ Fuel & $-4.24 \%$ & $-4.24 \%$ & $-2.73 \%$ & $-1.76 \%$ \\
$\Delta$ Cost & $-2.11 \%$ & $-1.85 \%$ & $-1.17 \%$ & $-0.84 \%$ \\
$\Delta$ Time & $2.18 \%$ & $2.12 \%$ & $1.43 \%$ & $0.73 \%$ \\
$\Delta$ Distance & $0.63 \%$ & $0.60 \%$ & $0.36 \%$ & $0.27 \%$ \\
\hline
\end{tabular}

Table 7. The minimum cost SAA formation flight compressibility study results. 


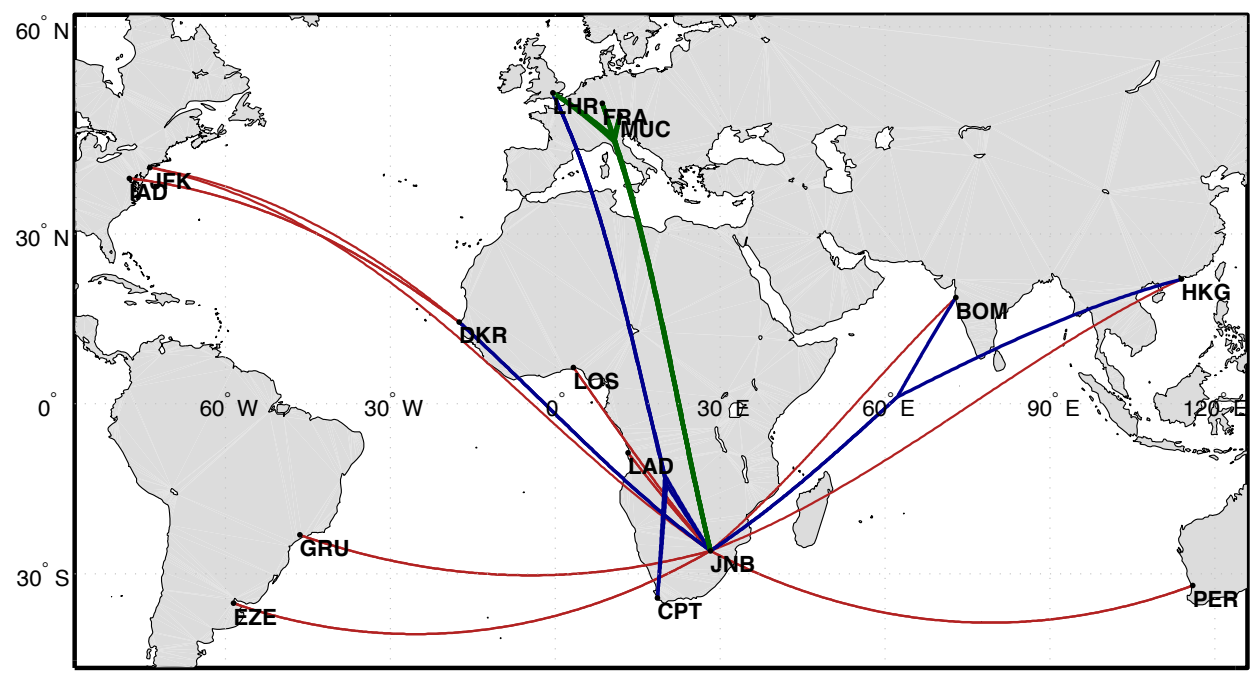

Figure 10. The minimum DOC SAA formation network with a $1 \%$ reduction in the maximum allowable formation cruise Mach number and a tip separation of $0.05 \mathrm{~b}$.

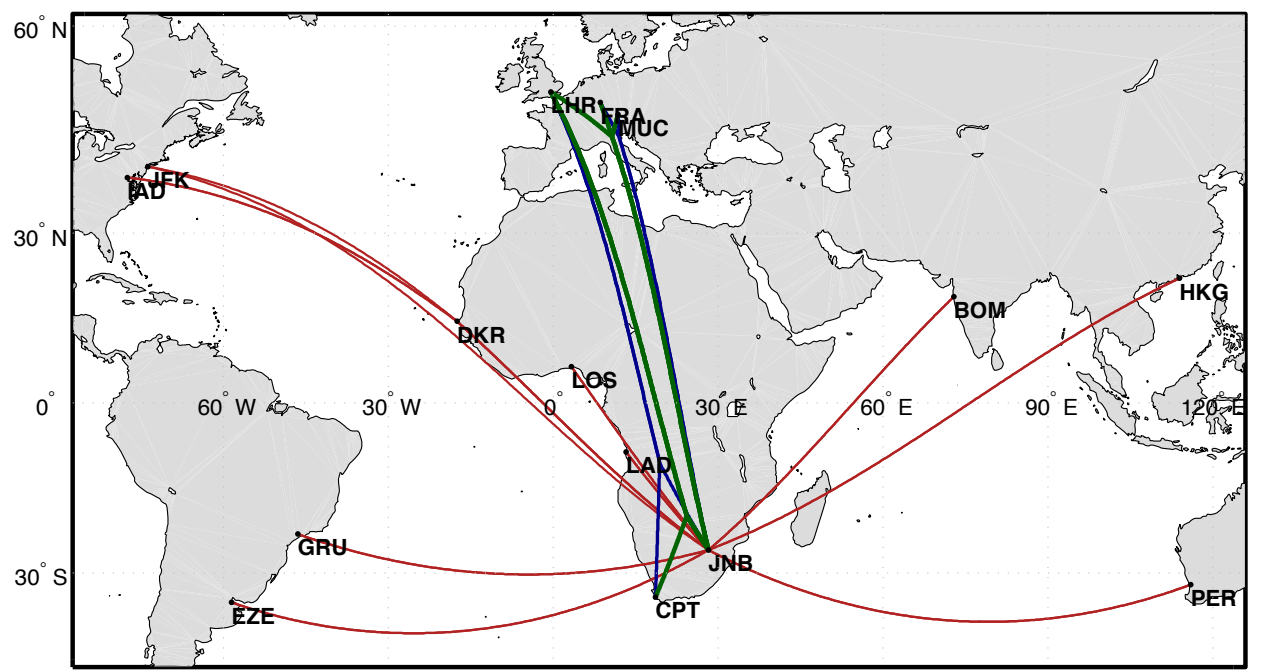

Figure 11. The minimum DOC SAA formation network with a tip separation of $0.10 b$.

\begin{tabular}{lllll}
\hline & Min. DOC & Case 1 & Case 2 & Case 3 \\
\hline Solo Flights & 13 & 13 & 17 & 21 \\
2-Aircraft Formations & 6 & 6 & 4 & 2 \\
3-Aircraft Formations & 2 & 2 & 2 & 2 \\
Distance in Formation & $49.7 \%$ & $49.2 \%$ & $39.8 \%$ & $31.8 \%$ \\
Time in Formation & $50.0 \%$ & $49.5 \%$ & $40.2 \%$ & $32.0 \%$ \\
Average $\Delta$ Departure Time (minutes) & 32 & 33 & 31 & 31 \\
\hline
\end{tabular}

Table 8. SAA formation compressibility study analytics. 


\section{Star Alliance Formation Flight Study}

The SAA network design results highlight the basic performance sensitivities associated with formation flight. An airline-by-airline implementation of formation flight is also more convenient initially given that the asymmetry in fuel savings between leading and trailing aircraft in formation can introduce cost-sharing issues among different airlines. There are also reasons however, to consider the formation flight benefits of large route networks that have multiple airlines plowing the same corridor. More planes flying similar routes should intuitively improve the viability of large formations and lead to increases network-wide savings.

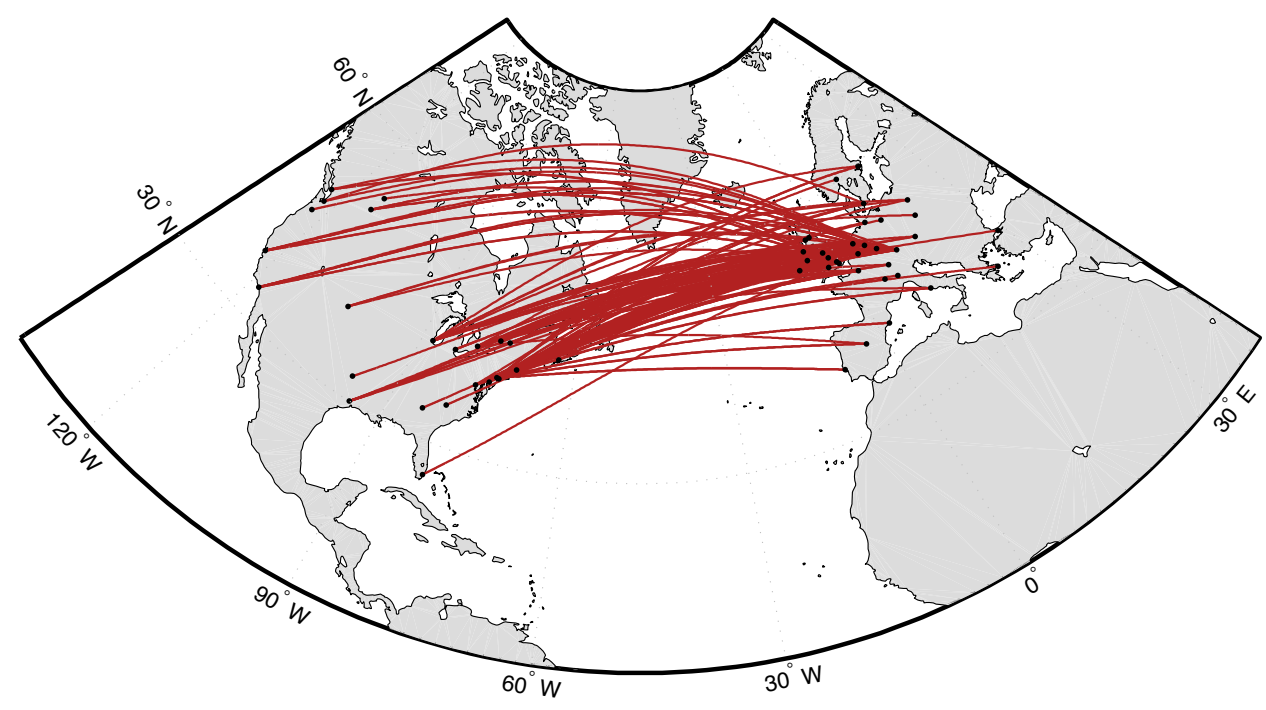

Figure 12. The Star Alliance transatlantic route network used in the design study.

Airline Alliances may be the logical place to start as it presents an organization structure to manage the cost-sharing associated with formation flight. In this section we consider a 150-flight snapshot of the eastbound Star Alliance transatlantic flight schedule. The route network is shown in Fig. 12. The schedule includes flights by 12 different types of aircraft in the Airbus A330, A340 and Boeing 737,747,757,767 and 777 families. An increase from 31 to 150 flights significantly increases the computational costs: for the transatlantic schedule there are 16,770 possible two-ship and 2,146,560 possible three-ship formations.

\begin{tabular}{ll}
\hline$\kappa_{r}$ & 0.15 \\
$\kappa_{t}$ & 0.48 \\
$r_{\text {mmax }}$ & $300 \mathrm{~nm}$ \\
$\Delta x$ & 20 spans \\
$\Delta y$ & 0 spans \\
$\Delta z$ & 0 spans \\
$\Delta \phi_{i j}$ & $30^{\circ}$ \\
$\Delta t_{a i}$ & 6 min \\
$\Delta t_{d i}$ & 6 min \\
\hline
\end{tabular}

Table 9. The Star Alliance heuristic filter and formation design parameters.

To render the problem tractable we impose the more restrictive filters in Tab. 9. Significantly, we decrease the departure and arrival flexibility from 1 hour in the SAA study to just 6 minutes. We also increase the required flight overlap requirement $k_{t}$ to $48 \%$ of the total scheduled flight time. Finally, we decrease the minimum formation aspect ratio parameter $\kappa_{r}$ discussed in Section II from 0.3 to 0.15. The more restrictive filters are justified by the large numbers of potentially good formations and narrow the scope of the subsequent mission optimization to the very best candidates. The heuristic filter removed $97.4 \%$ and $99.7 \%$ of all possible 
two and three-ship formations respectively. This still leaves a total of more than 800 formation missions to be optimized, which can take up to 60 CPU hours on a $2.1 \mathrm{GHz}$ AMD Opteron 6172 processor. The mission optimizations are naively parallel: every mission optimization is independent. A parallel implementation would allow us to scale the method to tackle large problems with relaxed heuristic filters.

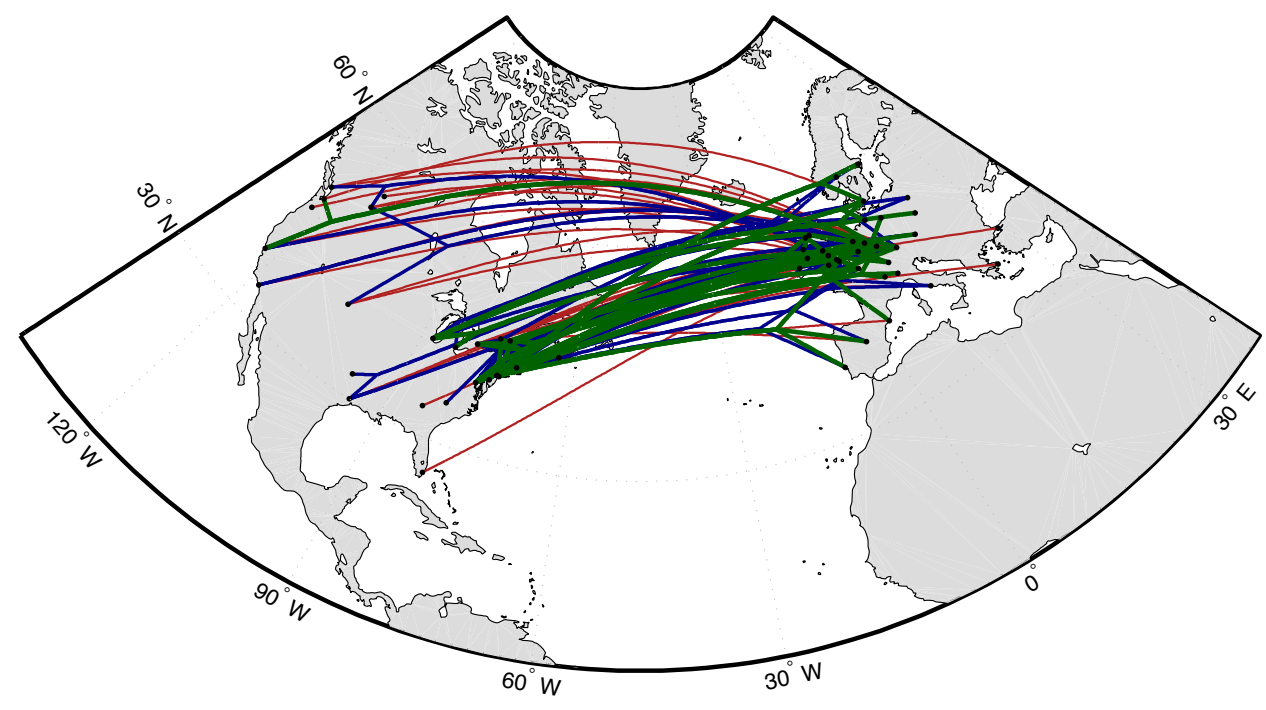

Figure 13. Star Alliance network optimized for minimum fuel formation flight. Two-aircraft formation segments are shown in blue. Three aircraft formations are in green.

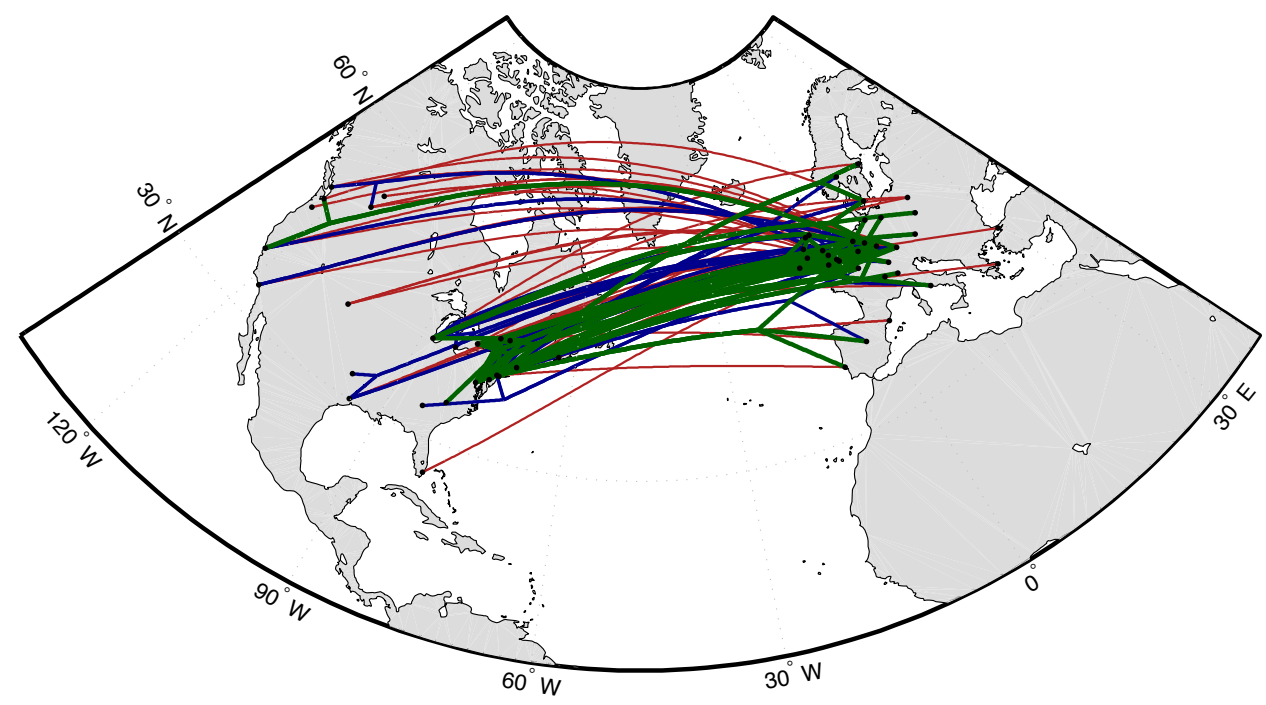

Figure 14. Star Alliance network optimized for minimum DOC formation flight.

Fig. 13 and 14 show the Star Alliance route network optimized for minimum fuel burn and cost. The results highlight the large number of viable three-ship formations in the minimum fuel network.

The results in Tab. 10 show that the minimum fuel formation network saves a significant $6.8 \%$ in fuel. The formation also sees a $2 \%$ cost reduction. The minimum DOC formation route network on the other hand saves $6 \%$ fuel and $2.4 \%$ cost against the corresponding solo network. The percent savings in both DOC and fuel have increased in all cases when compared with the smaller SAA network in Section VI. These savings are achieved with a restrictive departure and arrival flexibility of only 6 minutes. 


\begin{tabular}{lll}
\hline & Min. DOC & Min. Fuel \\
\hline$\Delta$ Fuel & $-5.99 \%$ & $-6.84 \%$ \\
$\Delta$ Cost & $-2.39 \%$ & $-2.01 \%$ \\
$\Delta$ Time & $3.70 \%$ & $6.2 \%$ \\
$\Delta$ Distance & $0.76 \%$ & $0.87 \%$ \\
\hline
\end{tabular}

Table 10. Star Alliance formation savings.

\begin{tabular}{lll}
\hline & Min. DOC & Min. Fuel \\
\hline Solo Routes & 48 & 33 \\
2-Aircraft Formations & 21 & 27 \\
3-Aircraft Formations & 20 & 21 \\
Distance Flown in Formation & $54.8 \%$ & $62.1 \%$ \\
Time Flown in Formation & $55.2 \%$ & $61.8 \%$ \\
Average $\Delta$ Departure Time & $4.9 \mathrm{~min}$ & $4.8 \mathrm{~min}$ \\
\hline
\end{tabular}

Table 11. Star Alliance formation network analytics.

Tab. 11 highlights structure of the optimized formation flight schedules. In each case the majority of the routes are flown in formation. The numbers of formation aircraft once again increase in the case of the minimum fuel formation. We observe that the large and spatially and temporally concentrated Star Alliance schedule is well-suited for formation flight.

\section{Conclusion}

In this paper we demonstrate a bi-level decomposition scheme to solve for the optimal formation flight schedule. The scale of the problem necessitates the careful development of heuristic search patterns to eliminate unlikely formations. The results of a series of design studies based on real-world route network and schedules demonstrate that formation flight can net significant fuel savings. The application of formation flight to a representative South African Airlines (SAA) long-haul route network can reduce fuel burn by over $4.6 \%$ or reduce direct operating cost by $2.1 \%$. The savings increase when aircraft from multiple airlines fly in similar corridors. The larger Star Alliance transatlantic route network can expect a $6.8 \%$ reduction in fuel burn or a $2.4 \%$ reduction in cost from optimized formation flight scheduling. Finally, the results of a preliminary study demonstrate that the formation flight network can be effectively designed at the system level to cope with compressibility effects induced by wake vortices.

The addition of a cost-sensitive design object sheds light on the economic viability of formation flight. We observe that aircraft must in general divert from their direct great circle route to meet up in formations. Although the drag savings from formation flight can more than make up for the fuel burn penalties from the diversions, the same cannot yet be said of the cost penalties stemming from the increased flight times. A more detailed cost model based on real world airline operations is needed to more accurately assess the cost impacts of formation flight.

\section{References}

${ }^{1}$ Wieselsberger, C., "Beitrag zur Erklärung des Winkelfluges einiger Zugvögel," Z. Flugtechnik $E$ S Motorluftschiffahrt, Vol. 5, 1914, pp. 225-229.

${ }^{2}$ Lissaman, P. B. S. and Shollenberger, C. A., "Formation Flight of Birds," Science, Vol. 168, No. 3934, 1970, pp. 1003, doi:10.1126/science.168.3934.1003.

${ }^{3}$ Hummel, D., "Aerodynamic Aspects of Formation Flight in Birds," Journal of Theoretical Biology, Vol. 104, No. 3, 1983, pp. 321-347.

${ }^{4}$ Weimerskirch, H., Martin, J., Clerquin, Y., Alexandre, P., and Jiraskova, S., "Energy Saving in Flight Formation," Nature, Vol. 413, No. 6857, 10 2001, pp. 697-698, doi:10.1038/35099670.

${ }^{5}$ Blake, W. and Multhopp, D., "Design, Performance and Modeling Considerations for Close Formation Flight," AIAA 
Atmospheric Flight Mechanics Conference and Exhibit, AIAA-1998-4343, August 1998, pp. 476-486.

${ }^{6}$ Frazier, J. W. and Gopalarathnam, A., "Optimum Downwash Behind Wings in Formation Flight," Journal of Aircraft, Vol. 40, No. 4, Feb 2003, pp. 799-803, doi:10.2514/2.3162.

${ }^{7}$ Wagner, E., Jacque, D., Blake, W., and Pachter, M., "Flight Test Results of Close Formation Flight for Fuel Savings," AIAA Atmospheric Flight Mechanics Conference and Exhibit, August 2002.

${ }^{8}$ Ray, R. J., Cobleigh, B. R., Vachon, M. J., and John, C. S., "Flight Test Techniques Used to Evaluate Performance Benefits During Formation Flight," TP-2002-210730, NASA, August 2002.

${ }^{9}$ Ning, S. A., Flanzer, T., and Kroo, I., "Aerodynamic Performance of Extended Formation Flight," Journal of Aircraft, Vol. 48, No. 3, May 2011, pp. 855-865, doi:10.2514/1.54636.

${ }^{10}$ Bower, G. and Kroo, I., "Multi-Objective Aircraft Optimization for Minimum Cost and Emissions Over Specific Route Networks," 26th Congress of International Council of the Aeronautical Sciences, Citeseer, 2008.

${ }^{11}$ Bower, G., Flanzer, T., and Kroo, I., "Formation Geometries and Route Optimization for Commercial Formation Flight," American Institute of Aeronautics and Astronautics, 1801 Alexander Bell Dr., Suite 500 Reston VA 20191-4344 USA, 2009.

${ }^{12}$ Liebeck, R. and Center, L. R., Advanced Subsonic Airplane Design \& Economic Studies, National Aeronautics and Space Administration, Lewis Research Center, 1995.

${ }^{13}$ Kroo, I., "An Interactive System for Aircraft Design and Optimization," Journal of Aircraft, Vol. 92-1190, 1992.

${ }^{14}$ McGeer, T. and Shevell, R., Method for estimating the compressibility drag of an airplane, Department of Aeronautics and Astronautics, Stanford University, 1983.

${ }^{15}$ Betz, A., "Behavior of Vortex Systems," TM-713, NACA, 1933.

${ }^{16}$ Delisi, D., Greene, G., Robins, R., Vicroy, D., and Wang, F., "Aircraft Wake Vortex Core Size Measurements," AIAA Applied Aerodynamics Conference, AIAA-2003-3811, June 2003.

${ }^{17}$ Holzäpfel, F., "Probabilistic Two-Phase Wake Vortex Decay and Transport Model," Journal of Aircraft, Vol. 40, No. 2, March 2003, pp. 323-331, doi:10.2514/2.3096.

${ }^{18} \mathrm{Ning}, \mathrm{S}$. A. and Kroo, I., "Compressibility Effects of Extended Formation Flight," AIAA Applied Aerodynamics Conference, June 2011

${ }^{19}$ King, R. M. and Gopalarathnam, A., "Ideal Aerodynamics of Ground Effect and Formation Flight," Journal of Aircraft, Vol. 42, No. 5, September 2005, pp. 1188-1199, doi:10.2514/1.10942.

${ }^{20}$ Ning, S. A., Aircraft Drag Reduction Through Extended Formation Flight, Ph.D. thesis, Stanford University, 2011. 